\title{
Interrelationships between cellulase activity and cellulose particle morphology
}

Olsen, Johan Pelck; Donohoe, Bryon S.; Borch, Kim; Westh, Peter; Resch, Michael G.

\section{Published in:}

Cellulose

DOI:

10.1007/s10570-016-0979-x

Publication date:

2016

Document Version

Peer reviewed version

Citation for published version (APA):

Olsen, J. P., Donohoe, B. S., Borch, K., Westh, P., \& Resch, M. G. (2016). Interrelationships between cellulase activity and cellulose particle morphology. Cellulose, 23(4), 2349-2361. https://doi.org/10.1007/s10570-0160979-x

\section{General rights}

Copyright and moral rights for the publications made accessible in the public portal are retained by the authors and/or other copyright owners and it is a condition of accessing publications that users recognise and abide by the legal requirements associated with these rights.

- Users may download and print one copy of any publication from the public portal for the purpose of private study or research.

- You may not further distribute the material or use it for any profit-making activity or commercial gain.

- You may freely distribute the URL identifying the publication in the public portal.

\section{Take down policy}

If you believe that this document breaches copyright please contact rucforsk@kb.dk providing details, and we will remove access to the work immediately and investigate your claim. 


\title{
Interrelationships between cellulase activity and cellulose particle morphology
}

\author{
Johan P Olsen ${ }^{1}$, Bryon S. Donohoe ${ }^{2}$, Kim Borch ${ }^{3}$, Peter Westh ${ }^{1}$ and Michael G. Resch ${ }^{4 *}$ \\ ${ }^{1}$ Research unit for Functional Biomaterials, Department of Science, Systems and Models, \\ Roskilde University, DK-4000 Roskilde, Denmark \\ ${ }^{2}$ Biosciences Center, National Renewable Energy Laboratory, Golden, CO \\ ${ }^{3}$ Novozymes A/S, Krogshøjvej 36, DK-2880 Bagsværd, Denmark \\ ${ }^{4}$ National Bioenergy Center, National Renewable Energy Laboratory, Golden, CO
}

*Corresponding author: Michael G. Resch, michael.resch@nrel.gov, (303) 384-7854

\begin{abstract}
It is well documented that the enzymatic hydrolysis of cellulose follows a reaction pattern where an initial phase of relatively high activity is followed by a gradual slow-down over the entire course of the reaction. This phenomenon is not readily explained by conventional factors like substrate depletion, product inhibition or enzyme instability. It has been suggested that the underlying reason for the loss of enzyme activity is connected to the heterogeneous structure of cellulose, but so far attempts to establish quantitative measures of such a correlation remain speculative. Here, we have carried out an extensive microscopy study of Avicel particles during extended hydrolysis with Hypocrea jecorina cellobiohydrolase 1 (CBH1) and endoglucanase 1 and 3 (EG1 and EG3) alone and in mixtures. We have used differential interference contrast microscopy (DICM) and transmission electron microscopy (TEM) to observe and quantify structural features at $\mu \mathrm{m}$ and $\mathrm{nm}$ resolution, respectively. We implemented a semi-automatic image analysis protocol, which allowed us to analyze almost 3000 individual micrographs comprising a total of more than 300,000 particles. From this analysis we estimated the temporal development of the accessible surface area throughout the reaction. We found that the number of particles and their size as well as the surface roughness contributed to surface area, and that within the investigated degree of conversion $(<30 \%)$ this measure correlated linearly with the rate of reaction. Based on this observation we argue that cellulose structure, specifically surface area and roughness, plays a major role in the ubiquitous rate loss observed for cellulases.
\end{abstract}

\section{Keywords}

Imaging, Transmission Electron Microscopy, cellulose surface structure, cellulase, cellobiohydrolase, endoglucanase 


\section{Introduction}

Essentially all reported experiments have shown that the enzymatic hydrolysis of cellulose exhibits a gradual loss of activity as the reaction progresses. This behavior is only partially explained by product inhibition, substrate depletion and physical instability of the enzymes (Zhang and Lynd 2004), and the underlying reasons for the ubiquitous slowdown has been the subject of much debate (Bansal et al. 2009). In the very early stage of enzymatic hydrolysis turnover slow-down might be explained by the processive nature of cellulases (Jalak and Väljamäe 2010; Praestgaard et al. 2011), and some reports have indicated that other enzyme effects like inactivation, unproductive binding or enzyme crowding might influence activity during extended hydrolysis as well (Eriksson et al. 2002; Yang et al. 2006). Nonetheless, most recent work has concluded, that the continued rate loss is mainly substrate dependent (Arantes and Saddler 2011; Bansal et al. 2012; Hong et al. 2007; Jeoh et al. 2007;

Luterbacher et al. 2015) even though relationships between substrate alterations and the reduced rate of hydrolysis remain poorly understood. Undoubtedly part of the rate decrease can be attributed to the heterogeneous nature of cellulose; in addition to being an insoluble polymer it is a mixture of amorphous and crystalline regions forming fibrils of various sizes depending on the cellulose source (Payne et al. 2015). This heterogeneity has been proposed to contribute to the observed rate loss. Thus it has often been suggested that the amorphous parts are degraded preferentially, leaving behind crystalline regions that are more recalcitrant towards degradation (Zhang and Lynd 2004), but reports that the crystallinity index of cellulose is unaffected by enzymatic degradation (Hall et al. 2010), has directed attention towards more continuous effects like changes in accessible surface area, pore size or available reactive sites on the substrate has also been proposed (Bansal et al. 2009; Grethlein 1985). Based on mechanistic models it has been suggested that change in substrate surface area is an important factor for the decline in hydrolysis rate (Levine et al. 2010). Hence, Bansal et al concluded that $90 \%$ of the rate decline is caused by a decrease in substrate accessibility and hydrolysability - a quantity the authors define as the fraction of enzyme binding sites that are available for hydrolysis (Bansal et al. 2012). 
Many studies have sought to clarify changes undergone by cellulosic substrates during hydrolysis using high resolution imaging techniques like atomic force microscopy (AFM), transmission or scanning electron microscopy (TEM, SEM) or fluorescence microscopy. Whole pretreated biomass has a complex architecture across multiple length scales, and while this architecture has been studied extensively for many different substrates (Antal 1985; Ciesielski et al. 2014; Donohoe and Resch 2015), only in rare cases have structural effects of enzymatic degradation of these substrates been reported (Resch et al. 2014). In contrast numerous studies on enzyme induced changes to the morphology of pure cellulose substrates like BMCC ribbons, Valonia fibrils and Avicel have been published over the years (for an extensive review see Bubner et al. 2013). The general observation is that cellobiohydrolases (CBHs) - notably CBH1 - target crystalline regions while endoglucanases (EGs) target amorphous regions, and that the combined action of the two types of enzymes has a profoundly different effect than each of them alone (Bubner et al. 2013; Payne et al. 2015). Furthermore it has been found that $\mathrm{CBHs}$ degrades cellulose fibrils from the ends and cause narrowing and sharpening of the fibril and indeed entire bundles of cellulose fibrils while EGs have no apparent spatial preference and cause general surface disruption or fibrillation (Chanzy and Henrissat 1985; Chanzy et al. 1983). Some studies have found that CBH1 acts to clear away sub-fibrils created by EG (Sprey and Bochem 1992) while others have observed that the action of $\mathrm{CBH} 1$ alone caused initial fibrillation, and only after extensive hydrolysis left behind thinned, sharpened and recalcitrant fractions (Chanzy et al. 1983; Imai et al. 1998; Jeoh et al. 2013). These studies have focused on structural changes mostly from a qualitative approach, but some attempts have been made at acquiring quantitative measures of changes in cellulose structure observed in AFM (Bubner et al. 2012; Wang et al. 2013) and fluorescence microscopy (Luterbacher et al. 2015) to elucidate the progression over time. These studies have provided valuable insights in the continuous morphological changes invoked by cellulases on cellulose substrates. However, to make continued observation of a defined area possible, the studied substrates were functionalized to a support material or solubilized and recrystallized to obtain a smooth surface. These modifications might affect the preferences for 
enzyme attack or alter structural integrity of the substrate and hence influence the morphological effects of enzymatic degradation. In addition the general approach is often incompatible with (or at least complicates interpretation of) bulk biochemical measurements of substrate conversion that are required for direct comparisons of substrate structure and activity. For these reasons it is not straightforward to draw conclusions from these studies regarding any effect substrate alterations might have on cellulase slow-down.

Here, we have used differential interference contrast microscopy (DICM) and transmission electron microscopy (TEM) to determine structural changes of Avicel particles at the $\mu \mathrm{m}-\mathrm{nm}$ scale during extended hydrolysis. The substrate was treated with two different endoglucanases (EG1 and EG3) and a cellobiohydrolase (CBH1) from Trichoderma reesei either alone or in mixtures. To get reliable, quantitative measures of the observed structural changes we implemented automated procedures for image analyses using the open source image processing software ImageJ. This allowed us to analyze a very large number of micrographs, which was necessary due to the heterogeneity of Avicel particles. In other words we did not attempt to identify distinct structural features in individual samples but rather to obtain quantitative ensemble average measures of structure changes analogous to what is obtained in biochemical activity measurements. This approach, along with the microscopy study being blinded, ensured an unbiased and reliable quantification of the morphological effect of cellulase activity on unmodified Avicel and allowed us to correlate these changes to the loss of hydrolytic activity.

\section{$\underline{\text { Materials and methods }}$}

\section{Cellulose digestion}

Unless otherwise noted, all chemicals were purchased from Sigma-Aldrich. All hydrolysis reactions were carried out in $30 \mathrm{mM}$ sodium acetate buffer, pH 5.0 with $2 \mathrm{mM} \mathrm{CaCl}_{2}$ and $0.01 \%$ sodium azide. Avicel PH 101 was washed by centrifugation, once in milliQ water and twice in reaction buffer. Final dry matter concentration in the reaction mixture was $10 \mathrm{mg} / \mathrm{mL}$ 
(1\% w/v). TrCel7A, TrCel7B and TrCel12A were heterologously expressed in Aspergillus oryzae and purified as described elsewhere (Westh et al. 2014). The enzymes were dosed at $100 \mathrm{mg} / \mathrm{g}$ Avicel either alone or in mixtures of $5 \%, 25 \%, 50 \%$ or $80 \%$ EG to total enzyme (see Table 1). Control samples were made without added enzyme. All samples were supplemented with $10 \mathrm{mg} / \mathrm{g} \beta$-glucosidase (Aspergillus niger) and incubated at $50^{\circ} \mathrm{C}$ with endover-end rotation at 10rpm. Samples were taken for activity measurements at $0,8,24,48,72$, and 96 hours. A subset of each sample from $0,8,48$ and 96 hours was stored at $-20^{\circ} \mathrm{C}$ for imaging. Glycan conversion was determined by HPLC as previously described (Resch et al. 2014). All image processing was carried out using Fiji, a distribution of the open source image processing software ImageJ (Schindelin et al. 2012). The analyses described below were written into macros to allow automatic processing.

Table 1 Enzyme composition in studied samples. Total enzyme dosage was 100mg/g Avicel. All samples were added $10 \% \beta$-glucosidase

\begin{tabular}{lcccccccccccc}
\hline & 1 & 2 & 3 & 4 & 5 & 6 & 7 & 8 & 9 & 10 & 11 & 12 \\
\hline CBH1 & $100 \%$ & $0 \%$ & $0 \%$ & $95 \%$ & $75 \%$ & $50 \%$ & $20 \%$ & $95 \%$ & $75 \%$ & $50 \%$ & $20 \%$ & $0 \%$ \\
EG1 & $0 \%$ & $100 \%$ & $0 \%$ & $5 \%$ & $25 \%$ & $50 \%$ & $80 \%$ & $0 \%$ & $0 \%$ & $0 \%$ & $0 \%$ & $0 \%$ \\
EG3 & $0 \%$ & $0 \%$ & $100 \%$ & $0 \%$ & $0 \%$ & $0 \%$ & $0 \%$ & $5 \%$ & $25 \%$ & $50 \%$ & $80 \%$ & $0 \%$ \\
\hline
\end{tabular}

Differential interference contrast microscopy:

Microscopy: Slurries of samples were transferred directly to a glass microscope slide and a cover slip was sealed around the edges to minimize evaporation. Images were taken with a SPOT RTKE CCD camera (Diagnostic Instruments, Sterling Heights, MI) on a Nikon C1 Plus microscope (Nikon, Tokyo, Japan) in bright field mode. All images were taken using a 60X 1.4 NA Plan Apo objective resulting in 1600 x1200 pixel images covering 195 x146 $\mu \mathrm{m}$ (corresponding to a pixel size of $122 \mathrm{~nm}$ ).

Image processing: Representative examples of DCIM micrographs before and after processing in ImageJ are shown in Figure 1 and a step-by-step walkthrough of the processing is shown in supplementary Figure S1. Initially, the contrast was enhanced, and the 24-bit RGB image was converted to a binary mask by applying the "Yen" threshold method (Kapur 
et al. 1985). A shadow from uneven illumination of the samples distorted the image processing along the edges of many of the DICM micrographs. To exclude this from the analysis we removed the outermost 60 pixels along all four edges of the image. Since DICM enhances contrast many particles would appear as only a perimeter. To make particles solid we applied the "Fill Holes" command before the perimeter and area as well as the maximum and minimum Feret's diameter (longest and shortest possible distance between two parallel tangents to the particle's perimeter, here used as a measure of particle length and width, respectively) was determined for each particle using Fiji's “Analyze Particles" function.

\section{Transmission electron microscopy}

Microscopy: $3 \mu 1$ sample was drop cast onto a 200 mesh carbon coated copper grid, negatively stained with $2 \%$ uranyl acetate and rinsed with water. The grids were imaged on a FEI Tecnai G2 20 Twin 200 kV LaB6 TEM (FEI, Hilsboro, OR) with a 4 megapixel Gatan UltraScan 1000 camera (Gatan, Pleasanton, CA). All images were acquired by the same operator to ensure consistent search criteria. To avoid bias the trial was blinded i.e. the operator was unaware which sample was on any particular grid. Grids were surveyed systematically at 1500x magnification and images were acquired at 3500x magnification, resulting in a frame size of $6.1 \times 6.1 \mu \mathrm{m}$. All particles large enough to be clearly identified at 1500x (more than $\sim 1 \mu \mathrm{m}$ long) and small enough to fit in the image frame at 3500x (less than $\sim 6 \mu \mathrm{m}$ ) were imaged. As evident from the results section, most often a large number of much smaller particles were included in the field of view. Approximately 80-90 images were captured for each sample.

Image processing and analysis: Noise was minimized by the "Remove Outliers" option, which changes the value of any pixel to the median of all pixel values in a surrounding block of designated size (here, 10x10 pixel) if that pixel deviates from that median by more than a specified value (1x standard deviation). Subsequently the images were converted from grayscale to a binary mask by applying the "Triangle" threshold method (Rogers et al. 1977) 
and the particle perimeters and areas were measured using the "Analyze Particles" procedure. Note, however, that even though holes are subtracted from particle area they are not included in the perimeter. Consequently, in images including overlapping particles these were assessed as single large particles with holes, the perimeter being only the outer perimeter of the particle. To overcome this we implemented a macro that first measured the area and perimeter of the particles and subsequently inverted the mask so as to measure the perimeter of the holes. The resulting two data files were combined with a script in Matlab (R2013a v 8.1.0.604, Natick, Ma, USA) to get the total perimeter and area. Thus the reported values are total perimeter and area for each image as opposed to each individual particle. A step-by-step review of the entire processing of a representative TEM micrograph can be found in the supplementary material (Figure S2).

\section{$\underline{\text { Results }}$}

12 separate Avicel samples were digested by either mono- or bicompononent enzyme solutions (see Table 1), and aliquots were taken out at 5 time points for biochemical analysis and 3 time points for imaging as described over. All samples had identical substrate and total enzyme loads at the beginning of the reaction. Five images were taken in the DICM for each sample at each time point. In the TEM 80-90 images were taken due to the much lower number of particles included in the image frame of this microscope at the magnification range used here. This resulted in 180 DICM and 2950 TEM micrographs, which were subjected to the semi-automated image analysis described over. 

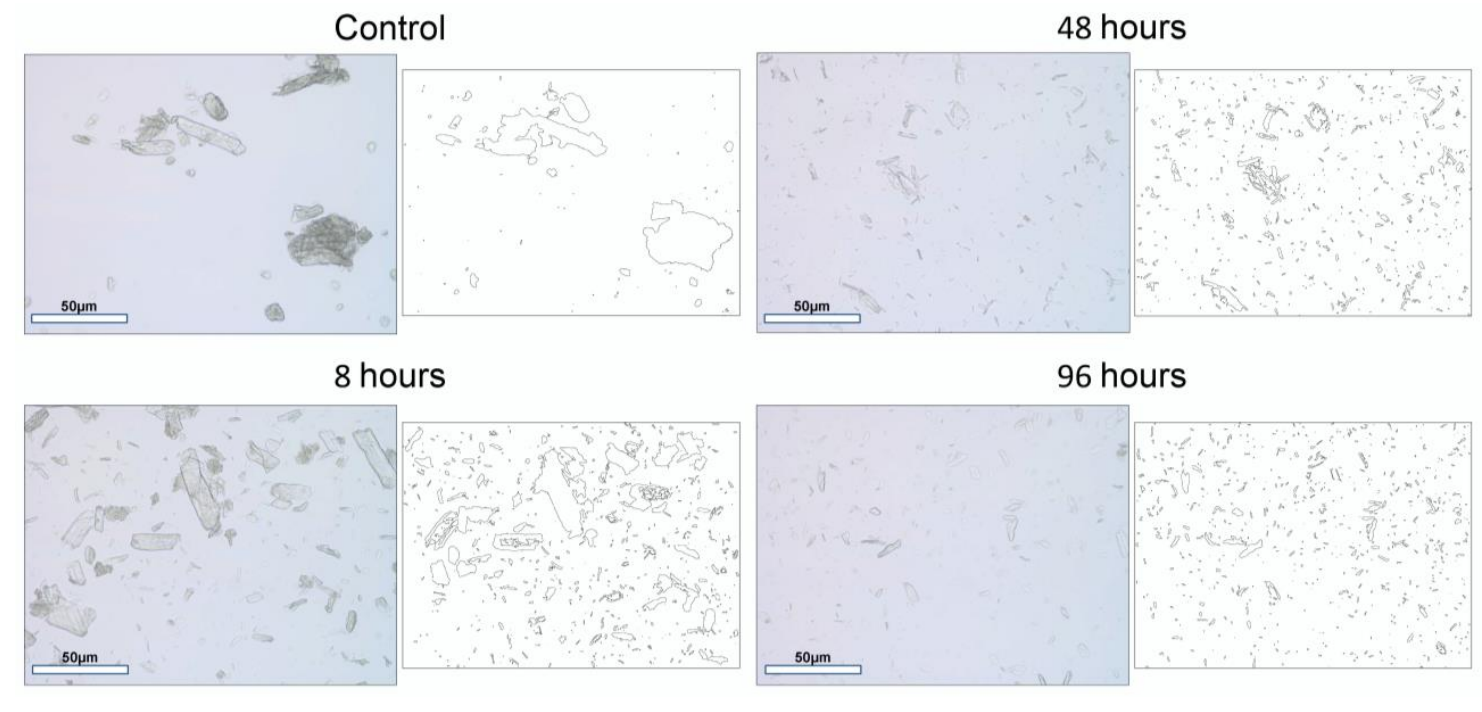

Figure 1 DICM micrographs of CBH1 digested Avicel after 8, 48 and 96 hours of

hydrolysis, plus control incubated without enzyme for 8 hours. For each sample, the raw micrograph is shown on the left. The outlines of all identified particles after trimming edges and excluding particles connected to the image frame is shown on the right (more details in Materials and methods). Scale bars $=\mathbf{5 0 \mu \mathrm { m }}$.

\section{Differential interference contrast microscopy}

The differential interference contrast microscopy (DICM) images covered a field of view of 195 x146 $\square$ m with a pixel size of $122 \mathrm{~nm}$, and a typical micrograph contain hundreds of particles ranging from a single pixel to $\sim 50 \square \mathrm{m}$ in length. Representative micrographs of CBH1 digested samples after 8, 48 and 96 hours of hydrolysis as well as an 8-hour control without added enzyme are shown in Figure 1. From visual inspection it is clear that the size distribution and number of particles change over time for this particular sample and similar trends were observed for all other samples (not shown). Generally we observed no major differences in the structural effects among different enzymes and mixtures; while different samples could often be distinguished from each other at a given time point, all samples followed similar trends when various structural parameters were considered in relation to the degree of conversion. We found that control samples without added enzyme contained considerably fewer particles than enzyme treated samples and that the number of observed 
particles increased with conversion at least in the initial phase (Figure 2, top). There was also a slight decrease in the mean particle size that is apparent in the representative micrographs shown in Figure 1. However, the measured lengths fell into a log-normal distribution, and as such the mean does not adequately describe the typical particle (supplementary material). If medians were considered instead of means neither the particle perimeter (not shown) nor the length (Figure 2, middle) changed with conversion. To determine whether the shape of the particles changed we computed their circularity. This size independent parameter, calculated as $4 \pi \cdot$ area/perimeter ${ }^{2}$, is a number between $0-1$ with 1 being a perfect circle. As seen in the bottom panel of Figure 2 the median particle circularity was also unrelated to conversion. This implies that the typical particle would not significantly shorten, narrow or any way change its general proportions on the $\square \mathrm{m}$ length scale within the conversion range studied here - an observation which will be addressed in more detail below.

One might expect that the typical particle size would decrease as more and more of the cellulose was solubilized, especially as this coincided with an increase in the number of particles. However, from a qualitative, visual survey of the micrographs it is apparent that a small number of relatively large particles (approximately $50 \square \mathrm{m}$ ) were present at low degrees of conversion but disappeared as the reaction progressed (Figure 1). This very small population being rapidly broken into many sub-particles might explain why we observed a constant median length but a slightly decreasing mean length. 


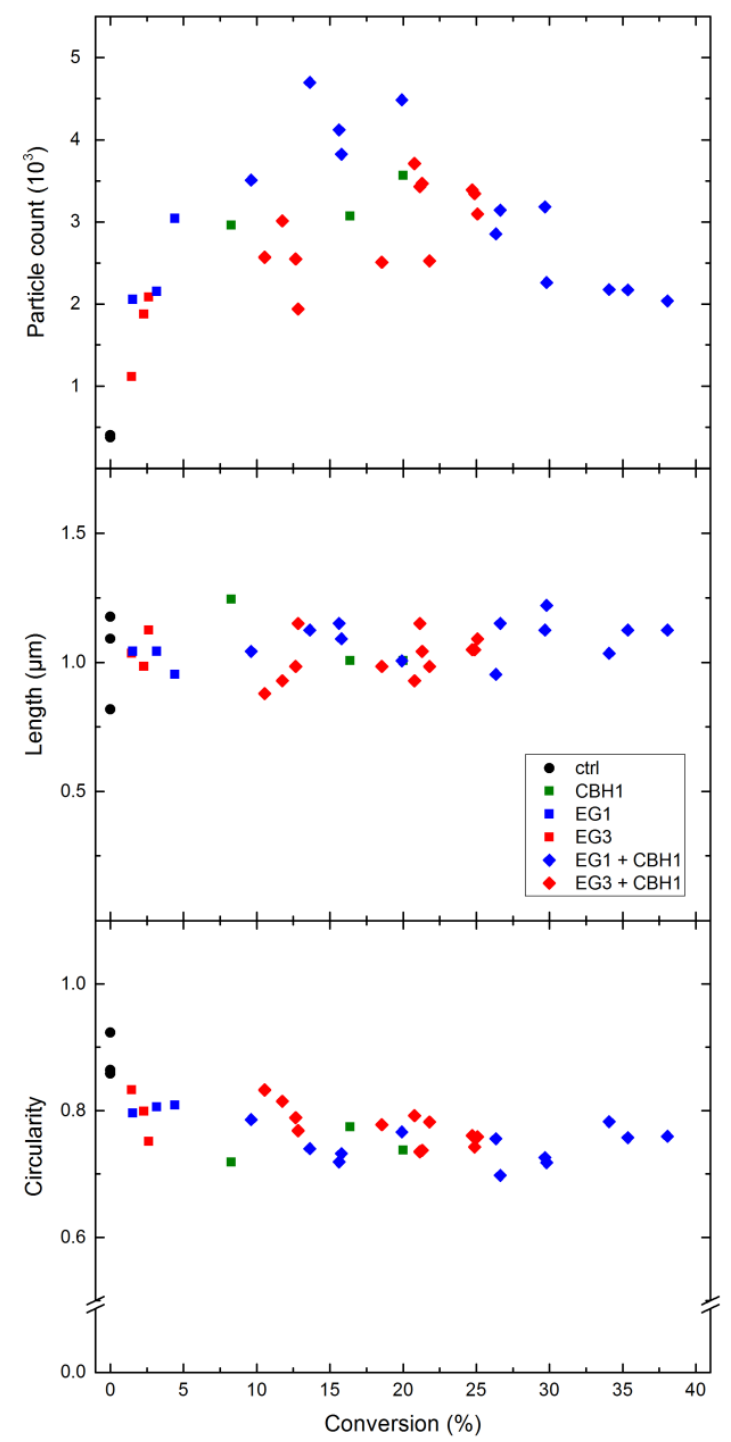

Figure 2 Results from automated analysis of DICM images plotted against conversion at 3 time points for 12 different digestions (control (black circles), CBH1 (green), EG1 (blue) and EG3 (red) alone (squares) or in 4 different mixing ratios (all represented by diamonds), see Table 1) Top: Total number of particles for each sample. Middle:

Median particle length determined as the Feret's diameter measured in ImageJ (see Materials and methods section). Bottom: Circularity (described in text)

\section{Transmission electron microscopy}

According to the DICM analysis the vast majority (>90\%) of the observed particles had a length of less than $5 \square \mathrm{m}$. When studying the samples in the TEM we observed that the 
particles from 1-5 $\mu \mathrm{m}$ exhibited very diverse morphologies. Many particles were surrounded by tiny fibrils or subparticles, some less than $10 \mathrm{~nm}$ wide but with a length of up to one $\mu \mathrm{m}$ and many of them overlapping to form relatively large aggregates (Figure 3). Some particles appear diffuse and still others appear condensed with a clearly defined perimeter (Figure 5B contains examples of all three morphologies). Importantly, no clear qualitative distinction between samples was possible because of this heterogeneity; all the investigated samples (including control samples without enzyme added) contained some examples of all particle morphologies and no systematic changes with time or enzyme mixture could be identified by visual inspection. As seen from Figure 3 the individual subparticles could not be distinguished in the automated analysis and for this reason we were unable to quantify structural parameters on a particle level as we did with DIC micrographs. Rather, for the TEM micrographs, we determined the total perimeter and area of every identified particle in a single micrograph. This resulted in 80-90 replicates (corresponding to the number of images) for each sample.
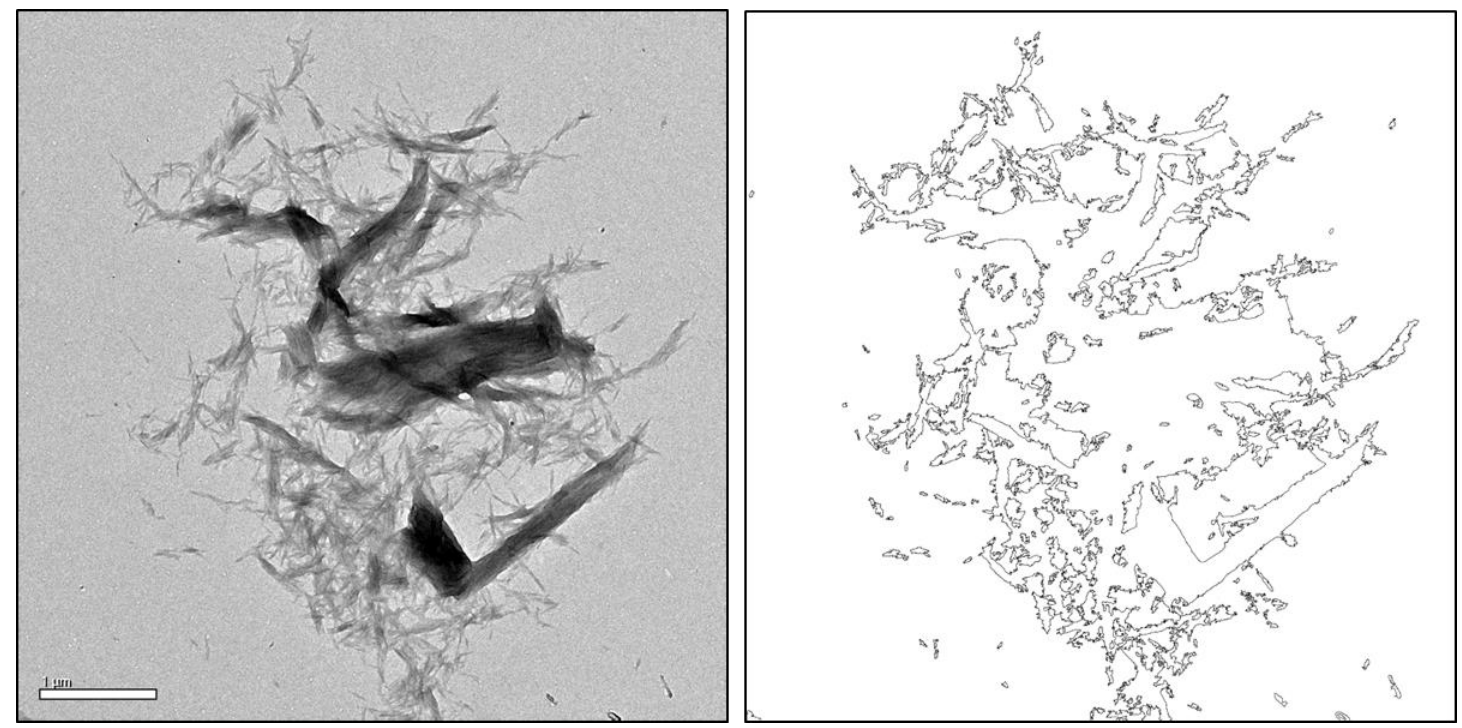

Figure 3 TEM micrograph of CBH1 digested Avicel after 8 hours of hydrolysis before image processing (left) and outlines of identified particles (right). Most sub-fibrils overlap making distinction between them impossible. Scale bar $=1 \square \mathrm{m}$. 


\section{Particle surface area and roughness}

Micrographs present a 2D projection of particles settled on a slide or a sample grid, and based on this we cannot directly determine 3D structural information. However the hydrolysis took place in bulk suspension before the particles settled on the imaging support. Unless there was a connection between enzyme target sites and how the cellulose particles settled on the support, the particles will be randomly oriented and the sizes and shapes of their $2 \mathrm{D}$ projection will presumably suffice as an approximation of the 3D surface parameters. We argue that this is the case in the present study even though a thorough exploration is beyond the current scope. Using the measured length and width of the particles observed in DICM and assuming a prolate spheroid shape we approximated apparent surface areas as follows

$$
A=2 \pi a^{2}+\frac{2 \pi a c}{e} \cdot \sin ^{-1}(e)
$$

where $c$ is half the measured length, a is half the measured width and $e$ is $\sqrt{c^{2}-a^{2}} \cdot c^{-1}$ (for more details see supplementary material)(Zwillinger 1996). The apparent surface area for each sample was calculated and as seen from Figure 4, this value increased during the first $\sim 10 \%$ conversion and then gradually decreased throughout the investigated conversion range. In contrast to the median values reported above - which gives information about the size and shape of the typical particle in a sample - the total surface area pr. micrograph is a relative measure of the "concentration" of substrate. With that in mind our results indicated that even though the $\square \mathrm{m}$ scale size and shape of a typical Avicel particle was similar throughout the reaction, the apparent total surface area in the sample did change with conversion. 


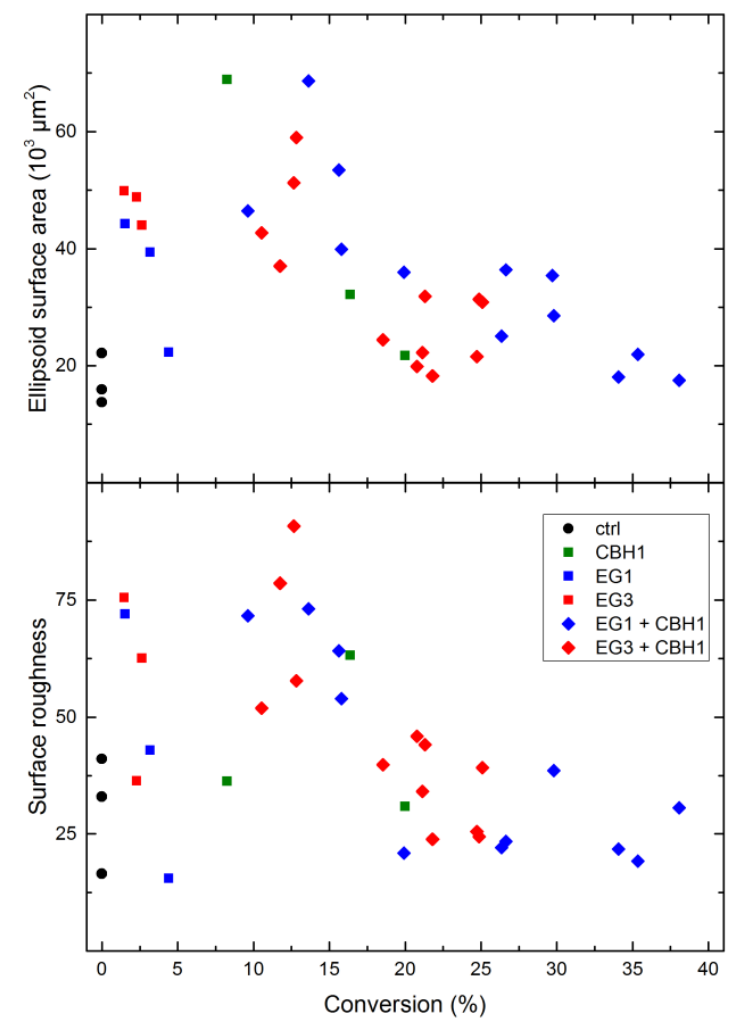

Figure 4 Total particle surface area calculated from DICM measurements (top) and mean surface roughness determined by TEM (bottom) plotted against degree of conversion. Both these measures increase during the first $\sim 10 \%$ conversion and then decrease as hydrolysis progresses. Symbols and colors have the same meaning as in Figure 3.

As described above, TEM revealed a high prevalence of composite structures comprising many small overlapping subparticles. We could not distinguish the individual subparticles in the automated analysis therefore we used the total perimeter and area in each micrograph to evaluate the relative surface roughness. We calculated the perimeter of a hypothetical circle with the same area as the total measured area for each micrograph (supplementary material). We then used the ratio of the observed perimeter to this theoretical perimeter as a relative measure of surface roughness on the nm scale. When plotted against overall substrate conversion (Figure 4) we observed a similar pattern to the approximated surface area calculated from DICM data; a slight initial increase followed by a marked decrease until it apparently leveled off at the highest conversions studied here $(\sim 30 \%)$. 


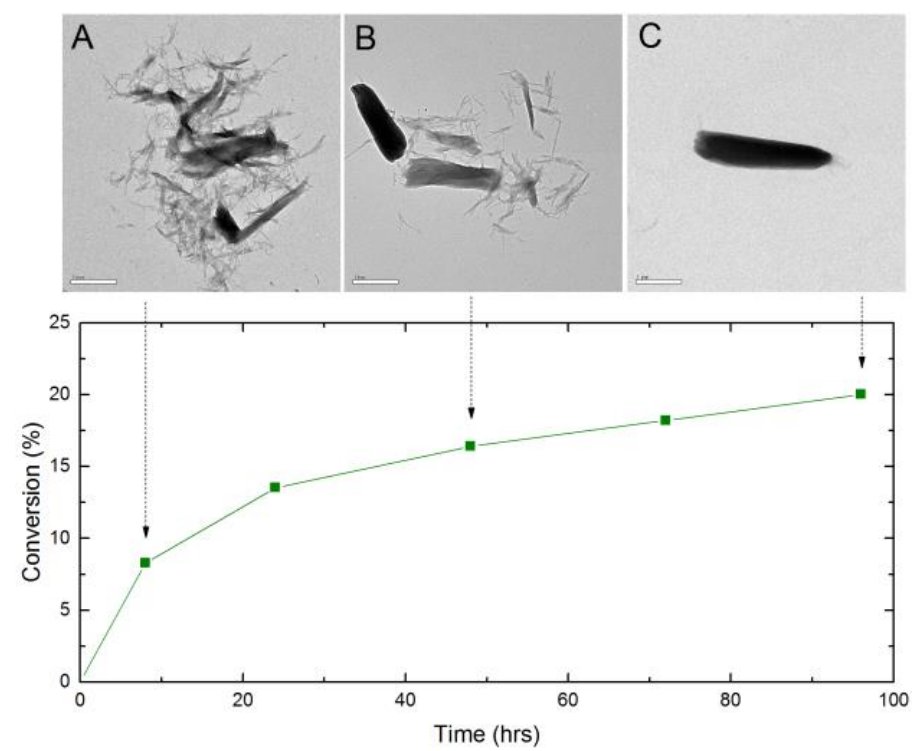

Figure 5 Suggested connections between particle structure and conversion. Top row shows TEM images of CBH1 digested Avicel after 8 (A), 48 (B) and 96 (C) hours. Bottom panel shows biochemical measurement of substrate conversion in the same sample over 96 hours. Different TEM micrographs of the any single sample were very diverse. Hence $\mathrm{A}, \mathrm{B}$ and $\mathrm{C}$ were selected to best represent the quantitative observations outlined in the text, i.e. that fibrillation decreased with hydrolysis time. Scale bars correspond to $1 \square \mathrm{m}$.

Figure 5 shows examples of TEM micrographs selected to represent the development in surface roughness with conversion. Based on the results of the automated analysis we suggest that sub- $\square \mathrm{m}$ fibrils are predominantly present in the early stages of the reaction. This would indicate that the actual surface area as experienced by an enzyme is decreasing in a manner, which is not readily observed on the length scale used in the DICM analysis. The relative surface roughness determined by TEM is a dimensionless number, equivalent to actual surface area (nm resolution) pr. approximated surface area ( $\square \mathrm{m}$ resolution). Thus we used the relative surface roughness determined by TEM to correct the surface area calculated from DICM measurements by multiplying these two values. The resulting parameter was used to approximate the accessible surface area within the studied samples. Plotting the rate of the 
reaction for $\mathrm{CBH} 1$ alone and in mixtures against this measure gave a reasonably linear correlation as seen in Figure 6.

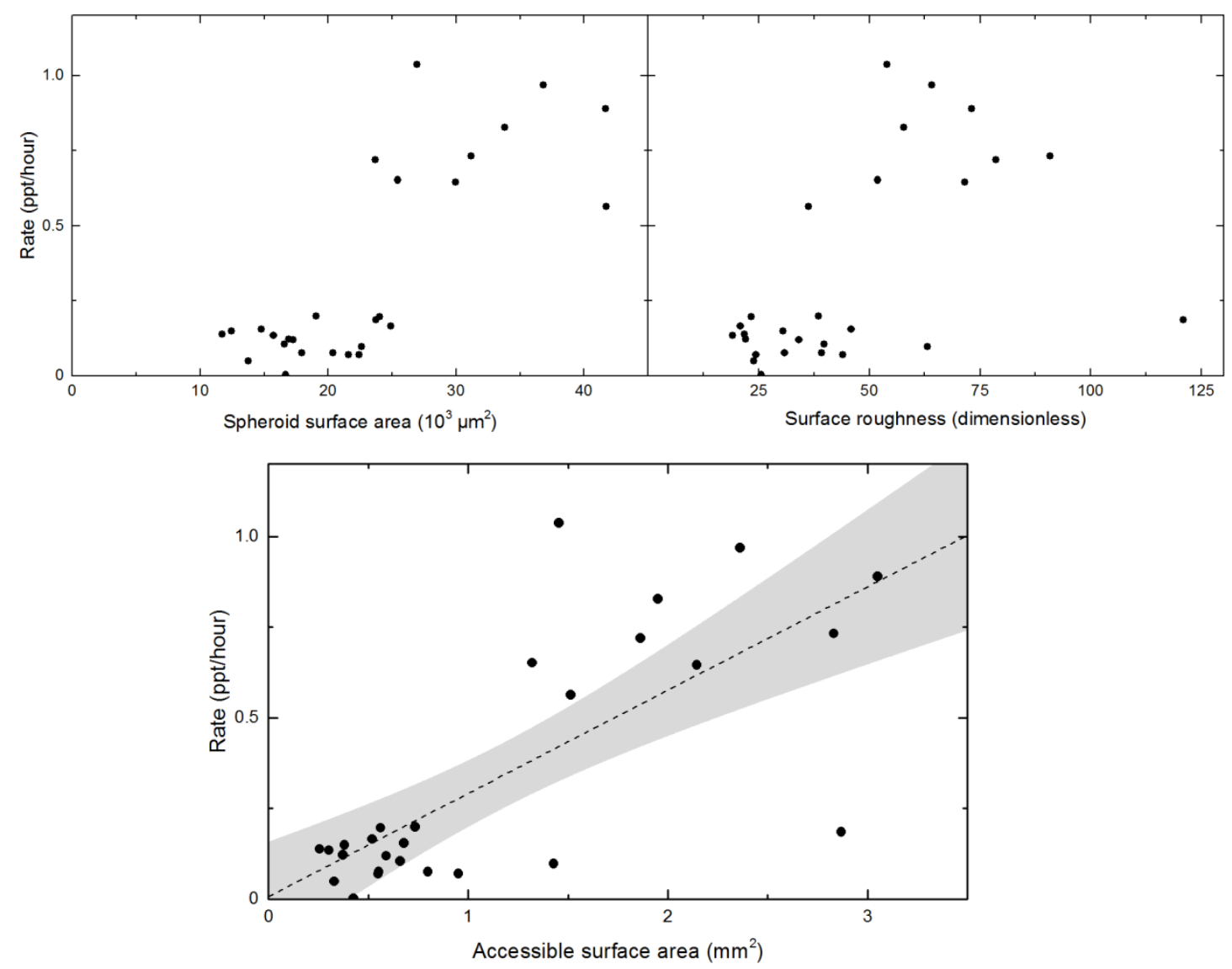

Figure 6 Reaction rate (percentage points per hour) plotted against calculated surface area determined by DICM (top, left) and surface roughness determined by TEM (top, right) as described in the text. The reaction rate in samples digested by $\mathrm{CBH} 1$ alone or in mixtures correlated linearly (dotted line) to the accessible surface area in the microscope field of view under the conditions studied here (bottom panel, 95\% confidence band shown as gray shade).

\section{Discussion and Conclusions}

We have used a combination of DICM and TEM to observe and measure changes in Avicel particle size and shape during enzymatic hydrolysis. We developed a method to perform a 
reliable and unbiased quantitation of size, shape and surface roughness of these particles on a $\mu \mathrm{m}-\mathrm{nm}$ scale. Based on analyses of 180 DICM and 2950 TEM micrographs comprising a total of more than 300,000 particles we found that for the enzymes studied here, the changes in shape and surface structure of Avicel was not determined by the type of enzyme or composition of enzyme mixtures but rather they depended on the degree of conversion that had taken place at the time of observation.

We observed a marked increase in particle count with conversion but no apparent change in the median size or shape of Avicel particles on a $\square \mathrm{m}$ scale. On a nm scale we observed a very large degree of heterogeneity in the surface structure of Avicel, but by increasing the sampling size we obtained reliable data on the surface roughness at this scale. When quantifying changes in relative surface area on both $\square \mathrm{m}$ and nm scale we found a slight initial increase followed by a continuous decrease throughout the studied range of conversion. Based on these observations we suggest that most particles were in the form of large superparticle aggregates prior to enzyme addition and that the initial activity of the studied enzymes rapidly broke these aggregates apart to form a large number of much smaller particles. In the following early phase of reaction a large fraction of the cellulose was in the form of sub- $\mu$ m fibrils that were difficult to distinguish by DICM. These fibrils appear to be relatively easy to degrade and their presence decreased as the hydrolysis progressed, leaving compact (possibly recalcitrant) particles behind in accordance with previous observations (Chanzy et al. 1983; Imai et al. 1998; Jeoh et al. 2013). By combining the DICM and TEM measurements we obtained a relative measure of the available surface area over the course of hydrolysis and, as was the case for the two values separately, this number initially increased slightly but subsequently decreased as the hydrolysis progressed to around $30 \%$ conversion where it leveled off. Interestingly, this is in agreement with the degree of conversion at which Bansal et al determined a leveling off of both accessibility and hydrolysability (Bansal et al. 2012). Furthermore we found that the rate of reaction for $\mathrm{CBH} 1$ alone and in mixtures was closely correlated to the relative accessible surface area in the conversion range studied here 
(Figure 6). Based on these findings we conclude that surface alterations on both $\square \mathrm{m}$ and nm length scales do indeed impact the rate loss that is unambiguously observed for cellulases in their hydrolysis of cellulose.

\section{Electronic supporting material available}

Supporting information includes details and examples on the automated image processing and analysis procedures for both DICM and TEM micrographs. Furthermore it includes details on the distribution of DICM data and on the geometry behind the conversion from 2D to 3D structural information.

\section{Acknowledgements}

This work was supported by the Danish Council for Strategic Research, Program Commission on Sustainable Energy and Environment (grant \# 11-116772 to P.W.), Augustinus Foundation (\# 14-4639 to JO) and Oticon Foundation (\# 14-3925 to JO). BSD was supported by the BioEnergy Science Center (BESC). BESC is a U.S. Department of Energy (DOE) Bioenergy Research Center supported by the Office of Biological and Environmental Research in the U.S. DOE Office of Science. MGR was supported by the DOE Office of Energy Efficiency and Renewable Energy, Bioenergy Technologies Office under Contract DE-AC36-08GO28308 with the National Renewable Energy Laboratory.

\section{Conflicts of Interest}

The authors declare that there are no conflicts of interest.

\section{$\underline{\text { References }}$}

Antal MJ, Jr. (1985) Biomass pyrolysis: A review of the literature part 1 - carbohydrate pyrolysis. In: Böer KW, Duffie JA (eds) Advances in solar energy. Springer New York, pp 61-111. doi:10.1007/978-1-4684-8992-7_3 
Arantes V, Saddler JN (2011) Cellulose accessibility limits the effectiveness of minimum cellulase loading on the efficient hydrolysis of pretreated lignocellulosic substrates. Biotechnology for Biofuels 4(3)

Bansal P, Hall M, Realff MJ, Lee JH, Bommarius AS (2009) Modeling cellulase kinetics on lignocellulosic substrates. Biotechnology Advances 27(6): 833-848

Bansal P, Vowell BJ, Hall M, Realff MJ, Lee JH, Bommarius AS (2012) Elucidation of cellulose accessibility, hydrolysability and reactivity as the major limitations in the enzymatic hydrolysis of cellulose. Bioresour Technol 107: 243-250

Bubner P, Dohr J, Plank H, Mayrhofer C, Nidetzky B (2012) Cellulases dig deep - in situ observation of the mesoscopic structural dynamics of enzymatic cellulose degradation. Journal of Biological Chemistry 287(4): 2759-2765

Bubner P, Plank H, Nidetzky B (2013) Visualizing cellulase activity. Biotechnology and bioengineering 110(6): 1529-1549

Chanzy H, Henrissat B (1985) Undirectional degradation of valonia cellulose microcrystals subjected to cellulase action. FEBS Letters 184(2): 285-288

Chanzy H, Henrissat B, Vuong R, Schülein M (1983) The action of 1,4- $\beta$-d-glucan cellobiohydrolase on valonia cellulose microcrystals. FEBS Letters 153(1): 113-118

Ciesielski PN, Wang W, Chen X, Vinzant TB, Tucker MP, Decker SR, Himmel ME, Johnson DK, Donohoe BS (2014) Effect of mechanical disruption on the effectiveness of three reactors used for dilute acid pretreatment of corn stover part 2: Morphological and structural substrate analysis. Biotechnol Biofuels 7: 47

Donohoe BS, Resch MG (2015) Mechanisms employed by cellulase systems to gain access through the complex architecture of lignocellulosic substrates. Curr Opin Chem Biol 29: $100-107$

Eriksson T, Karlsson J, Tjerneld F (2002) A model explaining declining rate in hydrolysis of lignocellulose substrates with cellobiohydrolase i (cel7a) and endoglucanase i (cel7b) of trichoderma reesei. Applied Biochemistry and Biotechnology 101: 41-60

Grethlein HE (1985) The effect of pore-size distribution on the rate of enzymatic-hydrolysis of cellulosic substrates. Bio-Technol 3(2): 155-160

Hall M, Bansal P, Lee JH, Realff MJ, Bommarius AS (2010) Cellulose crystallinity - a key predictor of the enzymatic hydrolysis rate. FEBS Journal 277(6): 12-12

Hong J, Ye X, Zhang YHP (2007) Quantitative determination of cellulose accessibility to cellulase based on adsorption of a nonhydrolytic fusion protein containing cbm and gfp with its applications. Langmuir : the ACS journal of surfaces and colloids 23(25): $12535-12540$

Imai T, Boisset C, Samejima M, Igarashi K, Sugiyama J (1998) Unidirectional processive action of cellobiohydrolase cel7a on valonia cellulose microcrystals. FEBS Letters 432(3): 113-116

Jalak J, Väljamäe P (2010) Mechanism of initial rapid rate retardation in cellobiohydrolase catalyzed cellulose hydrolysis. Biotechnology and Bioengineering 106(6): 871-883 
Jeoh T, Ishizawa CI, Davis MF, Himmel ME, Adney WS, Johnson DK (2007) Cellulase digestibility of pretreated biomass is limited by cellulose accessibility. Biotechnology and bioengineering 98(1): 112-122

Jeoh T, Santa-Maria MC, O’Dell PJ (2013) Assessing cellulose microfibrillar structure changes due to cellulase action. Carbohydrate Polymers 97(2): 581-586

Kapur JN, Sahoo PK, Wong AKC (1985) A new method for gray-level picture thresholding using the entropy of the histogram. Computer Vision, Graphics, and Image Processing 29(3): 273-285

Levine SE, Fox JM, Blanch HW, Clark DS (2010) A mechanistic model of the enzymatic hydrolysis of cellulose. Biotechnology and Bioengineering 107(1): 37-51

Luterbacher JS, Moran-Mirabal JM, Burkholder EW, Walker LP (2015) Modeling enzymatic hydrolysis of lignocellulosic substrates using confocal fluorescence microscopy i: Filter paper cellulose. Biotechnology and Bioengineering 112(1): 21-31

Payne CM, Knott BC, Mayes HB, Hansson H, Himmel ME, Sandgren M, Stahlberg J, Beckham GT (2015) Fungal cellulases. Chem Rev 115(3): 1308-1448

Praestgaard E, Elmerdahl J, Murphy L, Nymand S, McFarland KC, Borch K, Westh P (2011) A kinetic model for the burst phase of processive cellulases. FEBS Journal 278(9): $1547-1560$

Resch MG et al. (2014) Clean fractionation pretreatment reduces enzyme loadings for biomass saccharification and reveals the mechanism of free and cellulosomal enzyme synergy. ACS Sustainable Chemistry \& Engineering 2(6): 1377-1387

Rogers EW, Zack GW, Latt SA (1977) Automatic measurement of sister chromatid exchange frequenzy. The Journal of Histochemistry and Cytochemistry 25(7): 741-753

Schindelin J et al. (2012) Fiji: An open-source platform for biological-image analysis. Nat Methods 9(7): 676-682

Sprey B, Bochem H-P (1992) Effect of endoglucanase and cellobiohydrolase from trichoderma reesei on cellulose microfibril structure. FEMS Microbiology Letters 97(1-2): 113-117

Wang J, Quirk A, Lipkowski J, Dutcher JR, Clarke AJ (2013) Direct in situ observation of synergism between cellulolytic enzymes during the biodegradation of crystalline cellulose fibers. Langmuir : the ACS journal of surfaces and colloids 29(48): 1499715005

Westh P, Kari J, Olsen JP, Borch K, Jensen K, Krogh KBRM (2014) Cellobiohydrolase variants and polynucleotides encoding same. Patent no. WO 2014064115 A1

Yang B, Willies DM, Wyman CE (2006) Changes in the enzymatic hydrolysis rate of avicel cellulose with conversion. Biotechnology and Bioengineering 94(6): 1122-1128

Zhang YHP, Lynd LR (2004) Toward an aggregated understanding of enzymatic hydrolysis of cellulose: Noncomplexed cellulase systems. Biotechnology and Bioengineering 88(7): 797-824 
Zwillinger D (ed) (1996) Crc standard mathematical tables and formulae. 30th edn. CRCPress, Boca Raton, Florida 